\title{
Genetic investigation of the TSPYL1 gene in sudden infant death syndrome
}

Robert Hering, $\mathrm{MD}^{1}$, Rosario Frade-Martinez, MSc ${ }^{1}$, Thomas Bajanowski, MD ${ }^{2}$, Christian F. Poets, $\mathrm{MD}^{3}$, Frank Tschentscher, $M D^{2}$, and Olaf Riess, $M D^{1}$

\begin{abstract}
Background: Sudden infant death syndrome (SIDS) constitutes the most frequent cause of death in the postperinatal period in Germany. Recently, a lethal phenotype characterized by sudden infant death with dysgenesis of the testes syndrome (SIDDT) was identified to be caused by loss of function mutations in the TSPYL1 gene. Purpose: The study's purpose was to reveal a possible role of TSPYL1 in SIDS. Methods: DNA samples of 126 SIDS cases and 261 controls were investigated. Results: We found five sequence variations, each of them causing an amino acid substitution. No Hardy Weinberg disequilibrium and no significant difference in allele frequencies between patients and controls were observed for any variation. In one female patient a p. $\mathrm{F} 366 \mathrm{~L}$ amino acid polymorphism was found heterozygous, which could not be displayed in controls. A pathogenic implication of this substitution, which is conserved in primates and rodents, cannot be ruled out completely. Because SIDDT is the result of homozygous TSPYL1 mutations, this heterozygous exchange cannot solely explain the sudden death in this child. The reported mutation associated with SIDDT (457_458insG) was not detectable in our cohort. Conclusion: No association of sequence variations in the TSPYL1 gene and SIDS has been found in a German cohort. Genetic analysis of TSPYL1 seems to be of limited significance in the differential diagnosis of SIDS without dysgenesis of the testes. Genet Med 2006:8(1):55-58.
\end{abstract}

Key Words: TSPYL1, TSPYL, SIDS, SIDDT, sudden infant death syndrome

Sudden infant death syndrome (SIDS) is defined as the sudden death of an infant that remains unexplained after a careful autopsy, death scene investigation, and review of the medical history. ${ }^{1}$ With an incidence of 0.46 per 1000 life births in Germany in 2002, it constitutes the most frequent cause of death in the postperinatal period. ${ }^{2}$ Genetic (e.g., long QT syndrome genes, interleukin-10, and serotonin transporter gene) and environmental factors (e.g., prone sleep position, maternal smoking, and early weaning from breastfeeding) seem to contribute to SIDS. ${ }^{2}$ Recently, a lethal phenotype characterized by sudden infant death with dysgenesis of the testes (SIDDT) was identified to be caused by loss of function mutations in the TSPYL1 gene. ${ }^{3}$ To reveal a possible role of TSPYL1 in SIDS, we investigated DNA samples of 126 deceased children from the German study on sudden infant death (GeSID). ${ }^{4}$

\footnotetext{
From the ${ }^{1}$ University of Tuebingen, Institute for Human Genetics, Department of Medical Genetics, Tuebingen; ${ }^{2}$ University of Essen, Institute for Legal Medicine, Essen; ${ }^{3}$ University of Tuebingen, Department of Neonatology, Tuebingen.

Robert Hering, MD, Universität Tübingen, Institut für Humangenetik, Calwerstr. 7, 72072 Tübingen, Germany.

Received: May 3, 2005.

Accepted: October 31, 2005.
}

DOI: 10.1097/01.gim.0000195898.15290.03

\section{MATERIALS AND METHODS}

\section{Patients}

For the present study 126 consecutive cases from the GeSID, ${ }^{4}$ which were investigated between 1998 and 1999, were selected. A standardized autopsy protocol was applied in all 18 study centers involved. This standardized autopsy protocol is in accordance with the European guidelines for medico-legal autopsies ${ }^{5}$ and closely reflects the International Standardized Autopsy Protocol ${ }^{6}$ and protocols used in other studies on SIDS. ${ }^{7-9}$ The autopsy included a thorough external examination, a complete internal examination, extensive histology, neuropathology, a full analytic toxicology scheme, and microbiology and virology. Death scene investigation was performed, and the clinical history was analyzed. All 18 centers obtained the approval of their local medical ethics committees and the state data protection officer.

\section{Controls}

A total of 261 adults (aged 65-83 years, mean age $72 \pm 4.3$ years; $52 \%$ were males and $48 \%$ were females) from the general German population were investigated as control individuals.

\section{Mutation analysis}

By applying denaturing high-pressure liquid chromatography (dHPLC) technology, we screened the coding region of the TSPYL1 gene in five fragments (length 300-349 base pairs 
[bp]) for sequence variations. The TSPYL1 gene lacks introns and contains a coding region of $1314 \mathrm{bp}$ (GenBank accession no. AL050331), encoding a mature protein of 437 aa (GenBank XP_371844). Polymerase chain reaction (PCR) primers were constructed using the online software Primer3 (http://www. genome.wi.mit.edu/cgi-bin/primer/primer3_www.cgi) and the published genomic sequence of the TSPYL1 gene (Table 1). PCR was carried out in a thermocycler (GeneAmp PCR System 9700; Applied Biosystems, Foster City, CA) under standard conditions. Unpurified PCR products were analyzed by $\mathrm{dH}$ PLC analysis on the Wave DNA Fragment Analysis system (Transgenomic, Omaha, NE) at the column temperatures shown in Table 1. Column temperatures and running conditions were generated using the Wavemaker Software (Version 4.1.31, Transgenomic). Fragments 1-1 and 1-4 were screened at two different column temperatures to reach the most sensitive melting conditions spanning the entire PCR fragments. Subsequent direct sequencing of the suspicious PCR fragments was performed on a Beckman Coulter CEQ 8000 according to the manufacturer's recommendations (Beckman Coulter, Fullerton, CA). Nucleotide numbering uses the A of the ATG translation initiation start site as nucleotide +1 .

\section{Association study}

Frequencies of the TSPYL1 variations c.378G>T, c.528_529insGTG, and c.1098C $>A$ were investigated by $\mathrm{dH}-$ PLC. Each specific dHPLC heteroduplex signal was defined as heterozygous for the corresponding sequence variation. Samples with a homoduplex signal were pooled with a homozygous wild-type control to discriminate the homozygous wild type (homoduplex) from the homozygous sequence variation (heteroduplex). To investigate the frequency of the polymorphisms c.183C $>T$ and $c .220 G>C$ we performed restriction fragment length polymorphism analysis of fragment 1-1 using the restriction enzymes $H p h I$ and BamHI, respectively.

\section{Statistics}

The chi-square test was used to compare the observed distribution of each variant and the calculated expected distribu- tion under Hardy Weinberg equilibrium and to compare allele frequencies between patients and controls.

\section{RESULTS}

For the present study 126 SIDS cases ( 71 males/65 females) with a mean age at death of 140.7 days (standard deviation 85.3 days) were investigated (median 117 days, range 8-358 days).

With dHPLC and direct automated sequencing, five sequence variations in the coding region of the TSPYL1 gene were detected. Each of these variations causes an amino acid substitution. No significant Hardy Weinberg disequilibrium in patients and controls and no significant difference in allele frequency between patients and controls were observed for variation c.183C $>T$ (p.P62S), c.220G $>C$ (p.A74P), c.378G $>T$ (p.Q126H), and c.528_529insGTG (p.V176_L177insV) (Table 2). Furthermore, in one patient a p.F366L amino acid polymorphism (c.C1098A) was found heterozygous, which could not be displayed in the controls ( 522 chromosomes). This patient was born at term after an uneventful pregnancy (birth weight $3300 \mathrm{~g}$ ). She was found dead in a face-down position, covered with sweat, at 93 days of age. No pathologic findings were described in the autopsy protocol. Pertussis serology showed borderline results. Extensive histologic, toxicologic, virologic, and microbiologic investigations were negative. Because this patient was female, dysgenesis of the testes, the typical clinical features of SIDDT, could not be verified.

\section{DISCUSSION}

In our group of 126 patients with SIDS we found five sequence variations altering the amino acid composition of the encoded protein. Four of these, however, were found in similar frequencies in our control individuals. We exclude these polymorphisms as disease-causing mutations because three of them have been found homozygous at similar frequencies in patients and controls. It is also unlikely that the p.Q126H exchange is causative of SIDS because it was slightly more common in the control population. Furthermore, the amino acid

Table 1

Polymerase chain reaction and denaturing high-pressure liquid chromatography conditions used to screen the TSPYL1 gene for mutations

\begin{tabular}{|c|c|c|c|c|c|c|}
\hline Fragment & Primer sequence $\left(5^{\prime}-3^{\prime}\right)$ & $\begin{array}{l}\text { Fragment } \\
\text { size }\end{array}$ & $\begin{array}{l}\text { Annealing } \\
\text { temperature }\end{array}$ & $\begin{array}{l}\text { Column } \\
\text { temperature }\end{array}$ & Time shift & Gradient \%B \\
\hline $1-1$ & $\begin{array}{l}\text { AGTTCGGCCTCTGAGGAAA } \\
\text { CTTTGATCGCCACATGACC }\end{array}$ & $343 \mathrm{bp}$ & 60 & $\begin{array}{l}62 \\
64\end{array}$ & $\begin{array}{l}-0,5 \\
-0,3\end{array}$ & $\begin{array}{l}61-71 \\
60-70\end{array}$ \\
\hline $1-2$ & $\begin{array}{l}\text { TACTCCCCAGATCCGAGTTG } \\
\text { CCTTCCTTCACCACCTCAGC }\end{array}$ & $300 \mathrm{bp}$ & 60 & 63 & $-0,5$ & $60-70$ \\
\hline $1-3$ & $\begin{array}{l}\text { AAAGTGCGCCACCGTCTC } \\
\text { TAGTTCCTCCGCTCCAGGTA }\end{array}$ & $336 \mathrm{bp}$ & 60 & 62 & $-0,5$ & $61-71$ \\
\hline $1-4$ & $\begin{array}{l}\text { CAGCTGGAGCACAAGTTTGG } \\
\text { GAATGAAGGACTGGGGTCA }\end{array}$ & 349 bp & 60 & $\begin{array}{l}58 \\
60\end{array}$ & $\begin{array}{c}-0,5 \\
0\end{array}$ & $\begin{array}{l}61-71 \\
58-68\end{array}$ \\
\hline $1-5$ & $\begin{array}{l}\text { GAAACAAGCTGATTGTCAAGGA } \\
\text { GTGCAGGAGTATTCCCAAGG }\end{array}$ & 347 bp & 60 & 60 & 0 & $58-68$ \\
\hline
\end{tabular}

bp, base pair. 


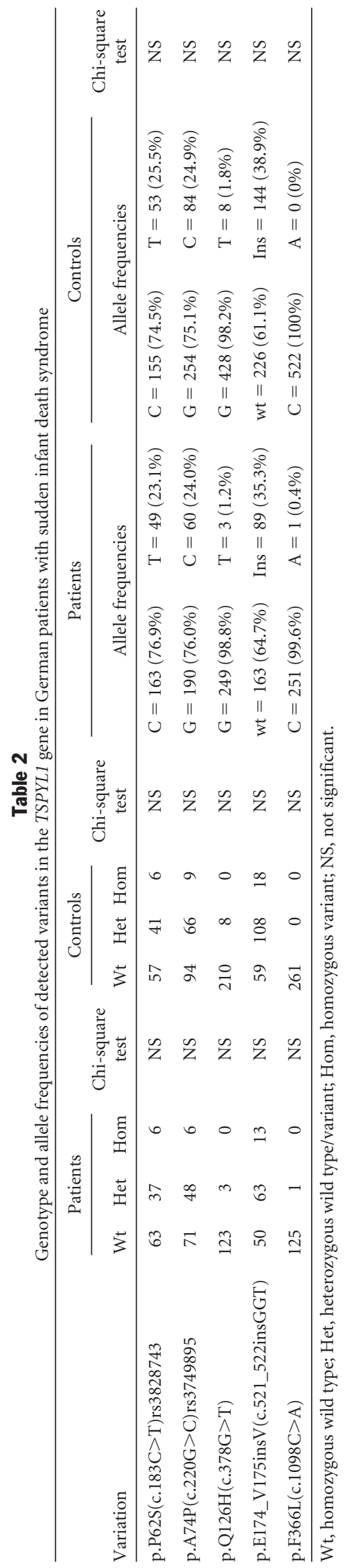

glutamine at position p.126Q is not conserved in rodents. Also, the $c .183 C>T$ (p.P62S, rs3828743) and c.220G $>C$ (p.A74P, rs3749895) polymorphisms are described in the National Center for Biotechnology Information databases in East Asians in frequencies of 0.586 and 0.631 , respectively, further indicating its low impact for SIDS.

Only one bp exchange, c.1098C $>$ A leading to a p.F366L substitution, was found exclusively in 1 of 126 patients, but not in any of our 261 controls. This amino acid is highly conserved in primates (Pan troglodytes, Pongo pygmaeus) and rodents (Rattus norvegicus, Mus musculus). After amplification of the complete coding region of the TSPYL1 gene (forward primer: AGTTCGGCCTCTGAGGAAA, reverse primer: GTGCAGGAGTATTCCCAAGG), the $c .1098 C>A$ substitution was also detected in a heterozygous state by direct sequencing. Thus, and because only one fragment could be displayed in the gel electrophoresis, larger deletions of parts of the coding region overlooked by dHPLC can be excluded.

Because SIDDT is caused by homozygous TSPYL1 mutations, this heterozygous exchange cannot explain the sudden death in this child. Because mutations in the promotor or intronic regions are not detected with the methods used in this study we cannot completely rule out the pathogenic implication of the $c .1098 \mathrm{C}>A$ substitution. We specifically looked for the 457_458insG mutation, which has been linked to the patients with SIDDT. ${ }^{3}$ However, this mutation was not detectable in our cohort.

\section{CONCLUSION}

TSPYL1 is a highly polymorphic gene. Mutations causing sudden death have not been found in this sample of German infants dying of SIDS. Thus, genetic analysis of TSPYL1 seems to be of limited significance in the differential diagnosis of SIDS among whites. However, TSPYL1 still needs to be investigated in other specific populations. Furthermore, because the identified polymorphisms were not associated with SIDS in our cohort, TSPYL1 variations are not suitable to predict SIDS or to specify the risk of recurrence in families with one affected child.

\section{ACKNOWLEDGMENTS}

The study was supported by the German Ministry of Science and Education (GeSID; $01 \mathrm{ED} 9401 / 8$ ). The authors thank the leaders of the GeSID group (B. Brinkmann, G. Jorch, and W. Köpcke) and all the members who performed the morphologic investigations of the cases and collected the epidemiologic data.

\section{References}

1. Krous HF, Beckwith JB, Byard RW, Rognum TO et al. Sudden infant death syndrome and unclassified sudden infant deaths: a definitional and diagnostic approach. Pediatrics 2004;114:234-238.

2. Bajanowski T, Poets CF. Der plötzliche Säuglingstod. Epidemiologie, Ätiologie, Pathophysiologie und Differentialdiagnostik. Dtsch Ärztebl 2004 2004;101:31853190 . 
3. Puffenberger EG, Hu-Lince D, Parod JM, Craig DW et al. Mapping of sudden infant death with dysgenesis of the testes syndrome (SIDDT) by a SNP genome scan and identification of TSPYL loss of function. Proc Natl Acad Sci U S A 2004;101:1168911694.

4. Findeisen M, Vennemann M, Brinkmann B, Ortmann C et al. German study on sudden infant death (GeSID): design, epidemiological and pathological profile. Int $J$ Legal Med 2004;118:163-169.

5. Brinkmann B. Harmonization of medico-legal autopsy rules. Committee of Ministers. Council of Europe. Int J Legal Med 1999;113:1-14.
6. Krous HF. Instruction and reference manual for the International Standardised Autopsy Protocol for sudden unexpected infant death. J SIDS Infant Mortality 1996;1:203-246.

7. Bajanowski T, Furst P, Wilmers K, Beike J et al. Dioxin in infants-an environmental hazard? Int J Legal Med 2002;116:27-32.

8. L'Hoir MP, Engelberts AC, van Well GT, Bajanowski T et al. Sudden unexpected death in infancy: epidemiologically determined risk factors related to pathological classification. Acta Paediatr 1998;87:1279-1287.

9. Mitchell EA, Scragg R, Stewart AW, Becroft DM et al. Results from the first year of the New Zealand cot death study. N Z Med J 1991;104:71-76. 